\title{
An Evaluation of Empirical Approach for Gearbox Diagnosis in the Construction Equipment
}

\author{
Keon $\operatorname{Kim}^{1}$ \\ ${ }^{1}$ Institute of Technology, Doosan Infracore Co., Ltd., Incheon, Republic of Korea \\ keon.kim2@doosan.com
}

\begin{abstract}
A planetary gearbox is one of the most important components in rotating machinery. In construction equipment, it is mainly used for swing and traveling devices due to huge torque capability and good torque transmission. Unexpected failure of the planetary gearbox can cause increased idle time, unnecessary cost due to inability of the construction site and safety hazards such as an operator isolation in a remote area. For these reasons, the need for timely prediction of unexpected failure becomes increasingly important in the field of construction machinery. In general, studies on fault diagnostic and condition monitoring of planetary gearboxes have been carried out in fields such as aircraft, wind-turbines and power plants. Researches in the above-mentioned fields usually require high performance computing power, and the burden of cost for diagnostic is relatively small. In addition, construction machinery also faces difficulties due to various uncertainties such as uncertain operating conditions that affect the vibration characteristics of gearboxes.
\end{abstract}

This study focuses on an approach to the vibration-based fault diagnostic methodology that can distinguish gear faults in a planetary gearbox in an excavator using vehicle-based test. First, we analyze the types of gear faults and the parts where failures occur mainly through the database of field failures. From this result, several fault types to be used in the experiment are selected, and an arbitrary fault is applied to gears. Secondly, since the vibration data is acquired directly from the excavator, the signal processing method that can remove the noise as much as possible and distinguish the fault is selected. Finally, optimized features are selected to minimize the uncertainty impacts that cannot be eliminated or unknown. Through this study, we confirmed the effect of the signal processing method which can be used in the planetary gearbox of the excavator as follows: 1) Several kinds of fault can be distinguished. 2) Faults and methodologies that can be distinguished in the constant speed

Keon Kim et al. This is an open-access article distributed under the terms of the Creative Commons Attribution 3.0 United States License, which permits unrestricted use, distribution, and reproduction in any medium, provided the original author and source are credited. condition 3) Faults and methodologies that can be distinguished in the transition speed condition.

\section{INTRODUCTION}

Traditionally, various studies have been carried out on the detection of normal operation or abnormal state by measuring the vibration of mechanical devices. Recently, the various methodologies for diagnostic and prognostic have been developed along with the remarkable improvement of the computing power. These studies have been conducted from the beginning in the aerospace and power plants where the burden of research and development cost is relatively small. In the field of mobility, application studies are actively conducted, centered on the automobile industry, where sales volume is large and technology maturity is high. In the field of construction equipment, global top tier makers are applying some of the huge mining equipment. Arakawa (2002) introduced the condition monitoring system of the mining equipment based on the operation data and $\mathrm{He}, \mathrm{He}$, and Zhu (2008) suggested the fault diagnostic method of the hydraulic systems in the excavator. Recently, many construction equipment manufacturers need to evaluate the applicability of diagnostic or prognostic technology that can be used in small or low-cost construction equipment and some applications are on the market.

This paper focused on an empirical approach to the evaluation of the planetary gearbox in construction equipment. The author performed feasibility project using a low cost sensor and several signal processing methodologies that can be used in a planetary gearbox of the construction equipment (Kim, 2017). The diagnostic flow-chart of signal processing methodology applicable to construction equipment is shown in Figure 1. The dashed line is for the above-mentioned preliminary study and is shown for the understanding of the process. First, sensors(i.e., accelerometer and tacho-meter) are attached to the gearbox of the excavator, and the vibration signal is acquired. The applied methodology is a total of three signal processing methodologies. After selecting the features for the detection 
of the fault with three signal processing methodologies, the optimized feature(i.e.,health index) is extracted through the optimization process considering the specificity of the excavator.

In this paper, the main purpose is to verify the possibility that the signal processing method which is well known to distinguish faults in a planetary gearbox (rotor system of power plant or helicopter) is well applied to an excavator with mobility on the ground. To improve fault detection performance in the target system that are heavily influenced by the surrounding environment, a methodology for modifying acquired health indexes is proposed.

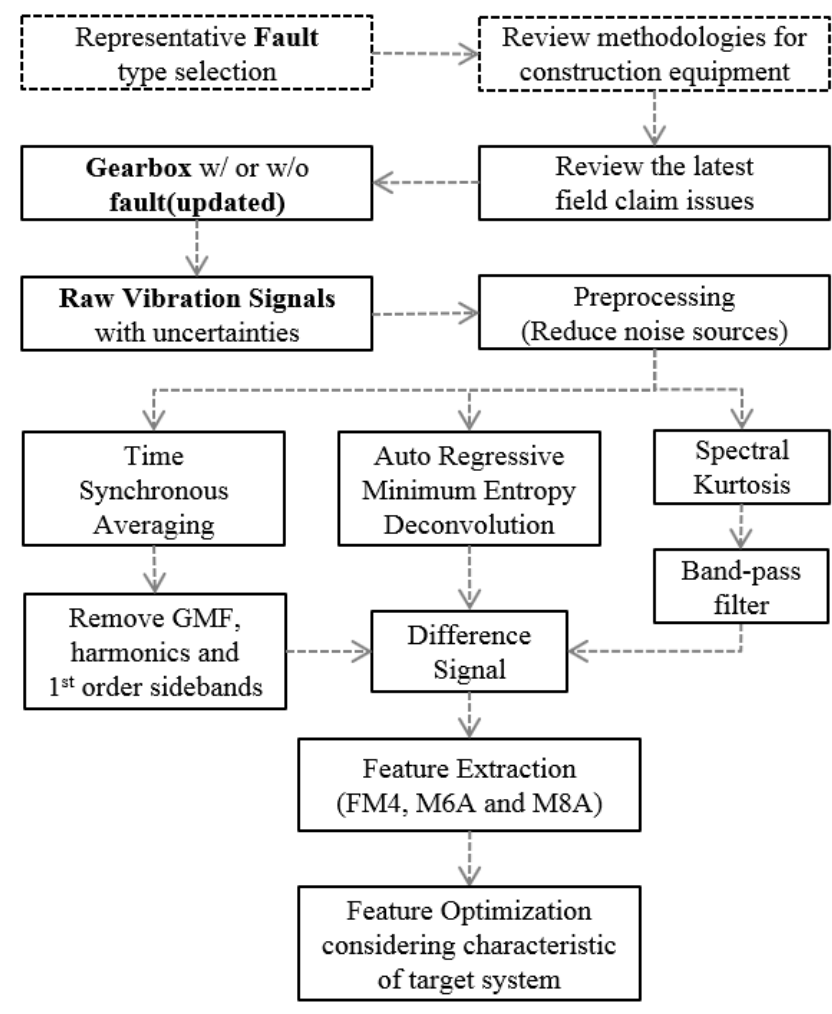

Figure 1. Diagnostic flow-chart for construction equipment.

\section{EXPERIMENT PREPARATION}

Experiments were conducted on the excavator as a typical construction machine. In the excavator, the reduction gear (i.e., planetary gear box) is mostly used for the swing operation, and in the caterpillar type, it is also used for traveling. Results of reviewing of fault cases and resources required for repair were comprehensively determined, and the reduction gear was selected as the diagnostic target system. After investigation the field claims, the types of faulty gear used for the experiment were selected. The test was carried out by measuring the vibration and tacho-signal by attaching an accelerometer, a tachometer, an amplifier, a power supply, and a DAQ to the vehicle. In order to obtain additional data for distinguishing between normal and fault, Information related to the operation of the equipment was collected at the same time as the vibration data acquisition period in the excavator controller unit.

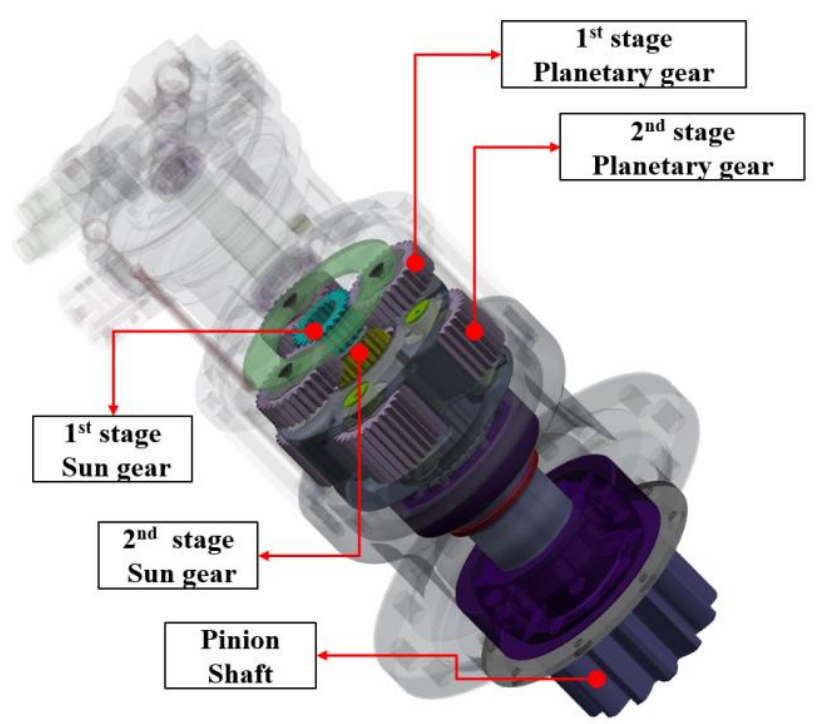

Figure 2. Schematic of the planetary gearbox in the construction equipment

\subsection{Representative Fault Selection}

The target system for the fault diagnostic was designated as the rotating machinery in consideration of the failure history, the severity of failure, and the difficulty of technology development, and the part was selected as the reduction gear through consultation and evaluation of the internal \& external expert group. In addition, a quadrant graph based on the frequency and the impact of failure proposed by Jay Lee et al. (2009) was referred. The diagnostic target item was decided to focus on the internal gear damage of the reduction gear through analysis of in-house failure cases, time required for repairs, and customer interviews. Trouble shooting cases in the field during the last several years for an excavator swing device, composed of a hydraulic motor and a planetary gearbox, were analyzed. In Figure 3, hundreds of cases are shown in percentage, and in gears and bearings of $60 \%$, most faults are identified as damage such as cracks, pitting, breakage and so on. The types of faults were identified using the research of Yongzuo el al. (2016). In that research, faults are divided into three types, and the frequency characteristics of each type are shown different. Among them, the faults of two types (i.e., impact and steady) except the compound type are selected. Table 1 shows the schematic and explanations for the arbitrary faults to be used in the test. In the subsequent diagnosis result, the fault type is denoted by I and S, and the fault type is denoted by TC, PF, S, ME, and W, respectively. For example, Normal would be $\mathrm{N}$, and impact type fault would be I-TC. 


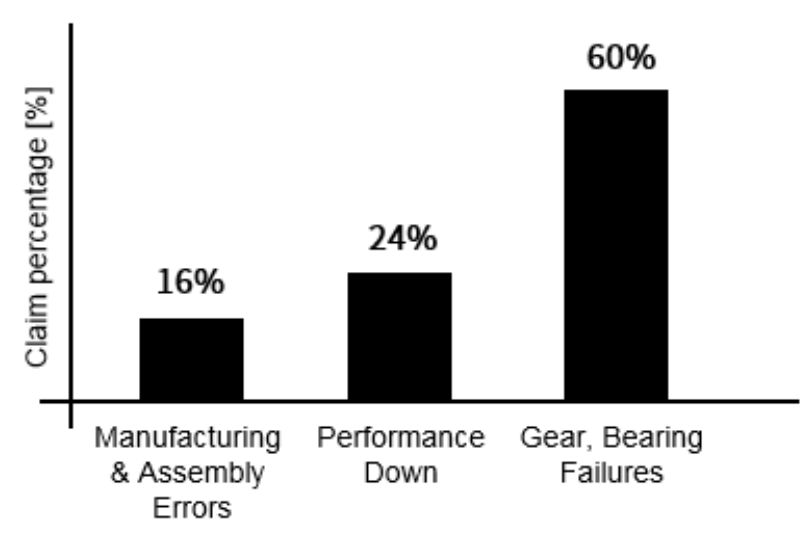

Figure 3. Classification of field claims of swing devices over the last several years

\subsection{Constraints and Prerequisites for the Vehicle Test}

Since the vibration-based diagnostic methods has been developed based on fixed or expensive equipment, the cost of accelerometers and equipment for data acquisition and analysis are not limited. However, in the technology development process, considering mass production, in the excavator, the constraints are relatively large, which is as follows:

1. It is difficult to install the tachometer to accurately measure the rotational speed of the rotating item in terms of technical difficulty and cost, and there is no place to utilize the information of the rotation for purposes other than diagnostic. That is, in the excavator position, it is only necessary to check whether the hydraulic fluid is flowing or not, and to check whether or not the hydraulic actuator is rotating.

2. The cost of sensors and additional devices is too high compared to the cost of the diagnostic target system. Optimization is needed to perform fault diagnostic with a minimum number of sensors and devices.

3. The performance of the computing module and the data storage space for the derivation of the health data are limited. Also, the communication cost between equipment and control system is a burden.

4. Because equipment can move and perform a wide variety of tasks, it is difficult to define a normal data cluster through continuous data collection.

Therefore, it is very important to use a minimal number of sensors and devices to acquire vibration signals and to perform a pre-test process to ensure the significance of data analysis. Prerequisites for vibration-based fault diagnostic in construction equipment are as below:

1. Cost-effective sensors to replace conventional tachometers and accelerometers should be available.

2. The fault diagnostic should be performed based on the computing power and data storage that can be utilized the most in the equipment. This should be taken into account, including the amount of dataset and communication costs needed to improve the performance of models and algorithms.

3. The frequency information of the vibration sources (i.e., noisy signals from multiple actuators in the excavator) other than the diagnostic target must be secured. It is also necessary to know the information about the deviations between excavators in the same model.

4. The representative operation mode based on the CUP (Customer Usage Profile) data of the equipment should be selected and validated.

Table 1. Representative faults and its type for the test

\begin{tabular}{|l|l|l|}
\hline Type & Representative fault & Schematic \\
\hline \multirow{5}{*}{ Impact } & $\begin{array}{l}\text { Tooth Crack } \\
\text { : Horizontal machining on } \\
\text { meshing plane }\end{array}$ & $\begin{array}{l}\text { Partial Fracture } \\
\text { Partial machining on the } \\
\text { upper surface }\end{array}$ \\
\cline { 2 - 3 } & $\begin{array}{l}\text { Spalling } \\
: \text { Large spall is machined } \\
\text { in the center of surface }\end{array}$ \\
\hline \multirow{5}{*}{ Steady } & $\begin{array}{l}\text { Manufacturing error } \\
\text { : Removal of the edge of } \\
\text { meshing plan }\end{array}$ & $\begin{array}{l}\text { Wear } \\
\text { : Removal of the entire } \\
\text { tooth surface with a certain } \\
\text { thickness }\end{array}$ \\
\hline
\end{tabular}

\subsection{Test Procedure}

The test begins by attaching sensors and devices to the excavator's swing device. The swing device consists of a hydraulic motor that converts the pressure of the hydraulic oil to the rotational force and a reduction gear that increases the force. The specification of swing device is shown in Table 2. The representative test mode was selected as the most used excavation and swing work in the excavator. Additional test parameters were set as RPM of the engine, weight (i.e., rotation inertia) of the excavator bucket, and slope of the work site. 
Table 2. Specification of the Swing Device

\begin{tabular}{|l|l|l|}
\hline & Item [Unit] & Value \\
\hline \multirow{3}{*}{$\begin{array}{l}\text { Swing } \\
\text { Motor }\end{array}$} & Max Speed [rpm] & 1,600 \\
\cline { 2 - 3 } & Output Torque [N-m] & 1,392 \\
\cline { 2 - 3 } & Displacement [cc/rev] & 278.8 \\
\hline \multirow{3}{*}{$\begin{array}{l}\text { *eduction } \\
\text { Gear }\end{array}$} & Gear Ratio [-] & 19.56 \\
\cline { 2 - 3 } & Max Speed [rpm] & 70 \\
\cline { 2 - 3 } & Output Torque [N-m] & 23,544 \\
\hline
\end{tabular}

*2-stage Planetary Gearbox

\section{GEARBOX DiagnOSTIC}

Chapter 3 discusses gearbox diagnostic. First, a pre-test is performed to identify various noise factors that may occur in the real vehicle data acquisition, to verify the availability of the acquired signal and to select the test mode. After performing the test as shown in Figure 4, examine the vibration signal to see if the fault signal is confirmed. If it is confirmed, the optimal signal processing method and the health data that can distinguish the normal and the failure are selected.
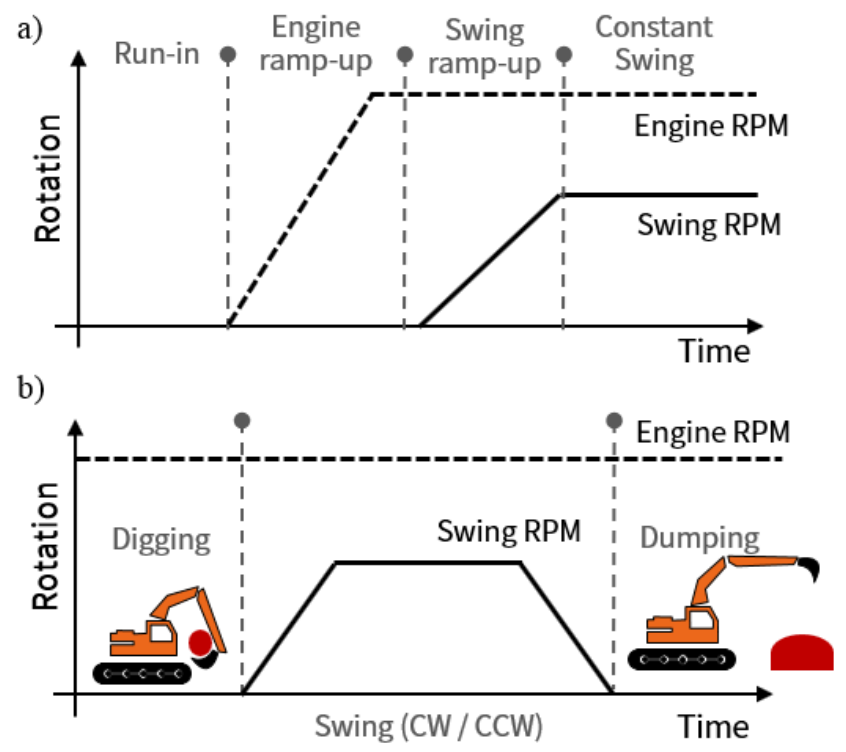

Figure 4. Duty cycle of the vehicle test, a) Constant speed condition; b) Excavator operation mode

Details of the test conditions are shown in Table 3. The 2nd sun gear was heavily loaded in the reducer, and the history that many fractures occurred in the field case was confirmed, and it was selected as the fault simulation gear. In the planetary gearbox, tooth meshing occurs simultaneously in sun gear, planetary gears and ring gear, and there is also a difference in meshing between each tooth. Assuming that the rotation starts in the stop state, the number of revolutions to the initial meshing condition is referred to as HTC (Hunting Tooth Cycle), and it is often used as a base period in time domain signal processing. The HTC of the target speed reducer is about 6 seconds for the first stage and about 25 seconds for the second stage.

Table 3. Design of Experiment

\begin{tabular}{|c|c|c|}
\hline Target gear & \multicolumn{2}{|c|}{$2^{\text {nd }}$ stage sun gear } \\
\hline $\begin{array}{l}5 \text { Fault } \\
\text { Samples }\end{array}$ & \multicolumn{2}{|c|}{$\begin{array}{l}\text { - Impact type : TC, PF, S } \\
\text { - Steady type : ME, W }\end{array}$} \\
\hline \multirow{2}{*}{$\begin{array}{l}\text { Input } \\
\text { speed }\end{array}$} & Engine & $1900 \mathrm{rpm}$ \\
\hline & Swing motor & $1150 \mathrm{rpm}$ \\
\hline \multirow{2}{*}{$\begin{array}{l}\text { Test } \\
\text { time }\end{array}$} & Constant speed & 60 seconds (30 cycles) \\
\hline & Transient speed & 20 seconds ( 10 cycles) \\
\hline Cycle time & \multicolumn{2}{|c|}{$\begin{array}{l}2 \text { seconds due to data storage in vehicle } \\
\text { condition }\end{array}$} \\
\hline
\end{tabular}

\subsection{Feasibility test}

The pre-test is similar to the run-in and ramp-up periods of Figure 4. a). After the start-up of the equipment, wait until the rotation, temperature, etc. of each part are saturated in the stabilization (i.e., run-in process) period. Engine RPM is gradually increased to the maximum value, and then each part is operated to check whether it is in normal operation, and only the swing operation of constant speed is performed separately. After the swing operation is completed, the test is terminated in the reverse order of the start procedure. Figure 5 shows the frequency components of each motion. The main prominent frequencies were identified as an engine, hydraulic motor, pump and so on. The following three additional variables of concern for diagnostics were found to be insignificant relative to vehicle level noises: 1) Differences of signals according to sensor installation position, 2) Posture of equipment (i.e., on flat ground or slope), 3) Load of excavator bucket when swing operation.

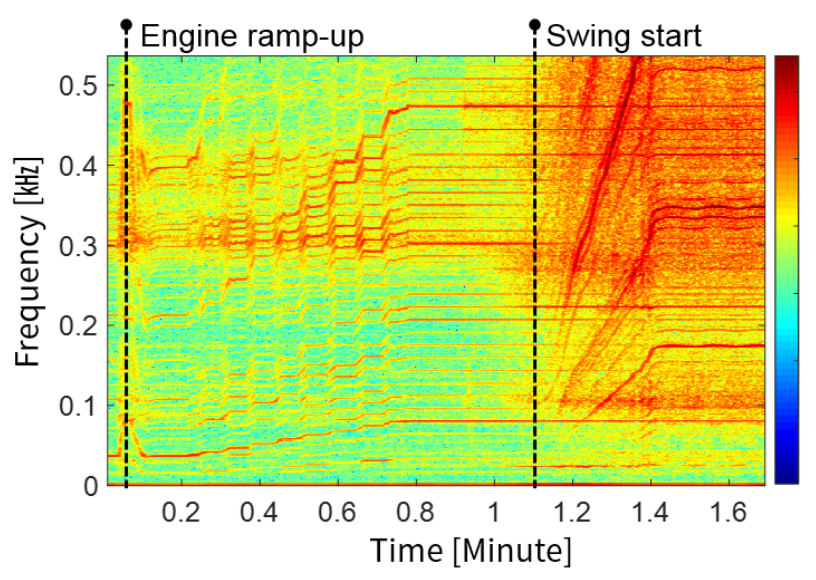

Figure 5. Spectrogram at the beginning of the pre-test 


\subsection{Signal processing-based method}

In this study, three signal processing methods are used to identify signs of failure: 1) Auto Regressive - Minimum Entropy Deconvolution (AR-MED) filter (Sawalhi, Randall, \& Endo, 2007) 2) Spectral Kurtosis (SK) (Antoni \& Randall, 2006) 3) Time Synchronous Averaging (TSA) (McFadden, 1987). The AR-MED filter serves to remove the repetitive signal from the AR filter and to highlight the peak of the MED filter. There is disadvantages that the signal is distorted due to the AR signal or a peak other than the peak due to the fault also emphasized. Spectral kurtosis is aimed at finding the region with the highest kurtosis in the entire frequency range, and sometimes domain knowledge of target system is needed for why the frequency range due to the fault is the corresponding range. Also, judging from the trial and error in the engineering process, it is difficult to diagnose the fault only by the value of kurtosis. Therefore, in this study, the highest kurtosis range was separated using a band-pass filter and used for feature extraction. The TSA averages the signal based on a constant number of rotations to remove noises and, in some cases, remove the Gear Meshing Frequency (GMF) and its sidebands components to help calculate features that are useful for fault diagnostic. However, since tachometer is essential, more sensors and additional devices are required than other signal processing methods. Each signal processing method is used to identify the fault signal in constant speed condition and equipment operation mode. The health data used FM4 (Zakrajsek et al, 1993), M6A and M8A (Martin, 1989) which were found to be effective in confirming the damage of the gear surface.

$$
\text { FM4, M6A and M8A }=\frac{N^{a} \sum_{i=1}^{N}\left(d_{i}-\bar{d}\right)^{2 *(a+1)}}{\left[\sum_{i=1}^{N}\left(d_{i}-\bar{d}\right)^{2}\right]^{(a+1)}}
$$

Where, $a$ is 1 for FM4, 2 for M6A and 3 for M8A. $d$ is the difference signal, which is the signal obtained by removing GMF, its harmonics and $1^{\text {st }}$ order sideband components from TSA-processed signal. Physically, it can be considered that the components related to noises and rotation are removed. In this study, processed signals from AR-MED and Spectral Kurtosis were used as difference signals to calculate health data. $\bar{d}$ is the mean value of the difference signal, and $N$ is the total number of data points in time record.

\subsubsection{Constant speed condition}

All of the three signal processing methods mentioned above were used to analyze the vibration data at constant speed condition. The distinction between normal and fault can be confirmed by checking the peak interval due to fault in the time domain signal, envelope analysis, frequency range of the difference signal, and so on. As a result of the frequency analysis of difference signal, most of the signal processing results confirmed the region around $7 \mathrm{kHz}$, but it is difficult to secure the reliability of the result because it is impossible to confirm the information about the natural frequency of the complex system. The envelope analysis identifies the frequency component due to the rotation of faulty gears, but it is not judged in a reliable way because the other components are more dominant. Figure 6 shows the sample period of raw vibration data and signal processing results for five faulty gears. a)

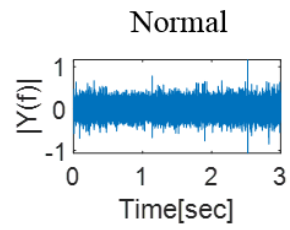

b)

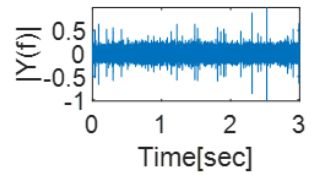

c)

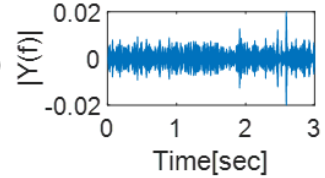

d)

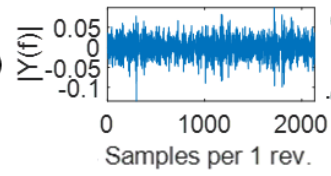

Tooth Crack
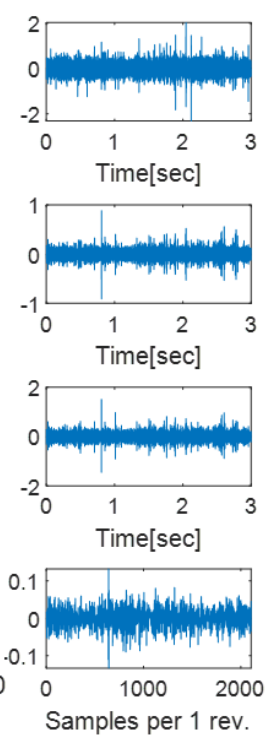

Partial Fracture
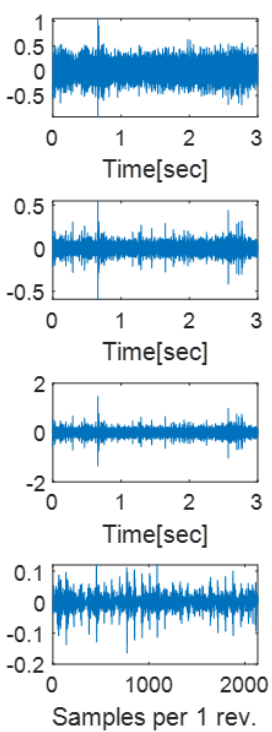

Spalling
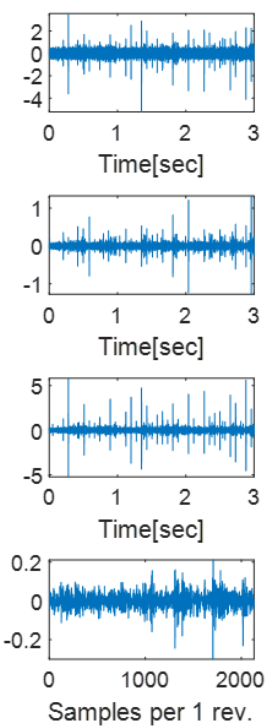
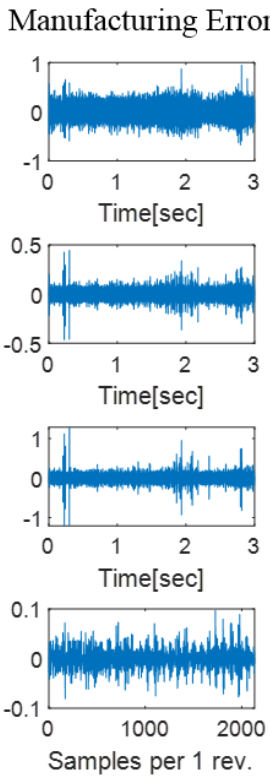

Wear
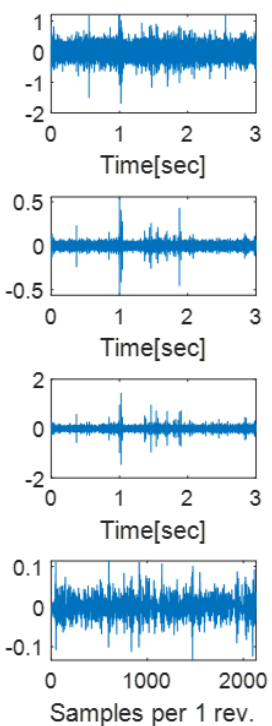

Figure 6. Time domain vibration data under various signal processing methods, a) raw signal; b) AR-MED filter; c) Spectral Kurtosis \& band-pass filter; d) TSA 
The M8A values are shown in Figure 7. Three kinds of health data, including M6A and M8A, derived from FM4, a concept of normalized Kurtosis, showed a similar tendency. The dotted line represents the threshold of the highest value among the normal state health data. In the case of some faulty gears, the value is smaller than the normal value. TSA has the lowest overall feature value, and as seen in the signalprocessed time domain signal, it is judged to refine the vibration data the most, but only two faults can be detected. AR-MED and Spectral Kurtosis did not detect one or two each, but the tendency of feature was found to be similar. It is judged by the influence of unreduced (or non-removable) factors in the vehicle test, not RIG-tester.

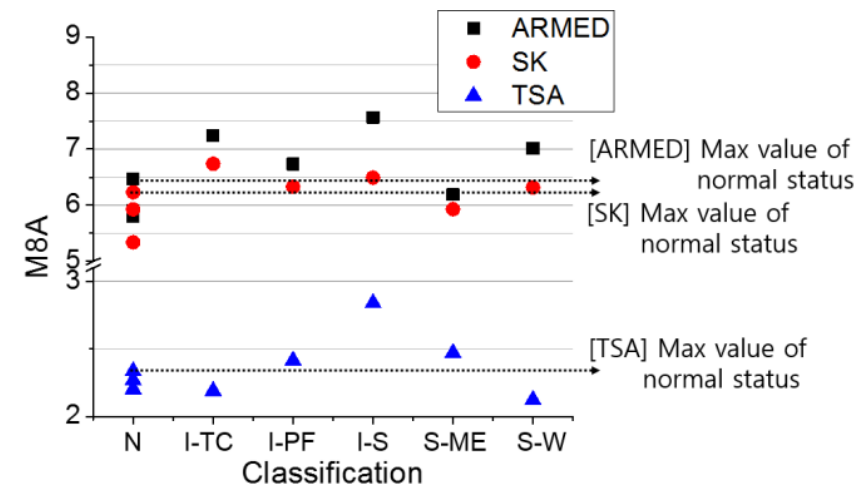

Figure 7. Average of M8A using TSA, ARMED and Spectral Kurtosis at constant speed condition

\subsubsection{Operation mode}

As a result of the pre-test, Spectral Kurtosls and AR-MED, which perform signal processing in the frequency domain, were found to have no distinction between faults in nonconstant speed cases. Spectral Kurtosis has not been identified in the frequency domain (except for the technical proof that the filtered frequency range best represents the fault frequency), and in the case of AR-MED, rather, distortion such as signal amplification occurred severely. The AR-MED filter is judged to be ineffective in removing the repetitive signal by using the AR model. Spectral Kurtosis has lost the strength of finding the frequency range with the highest kurtosis because of changes of frequency factor including GMF due to change of rotation speed. Therefore, only the TSA methodology is applied for fault diagnostic in the operation mode, and the results are shown in Figure 8. The method of TSA is divided into three periods of acceleration, constant speed, and deceleration by using the test mode in Figure 4. (b). As with the constant speed results, some failure cases showed smaller health data than normal, and most of the faulty gears were less than normal in the deceleration period

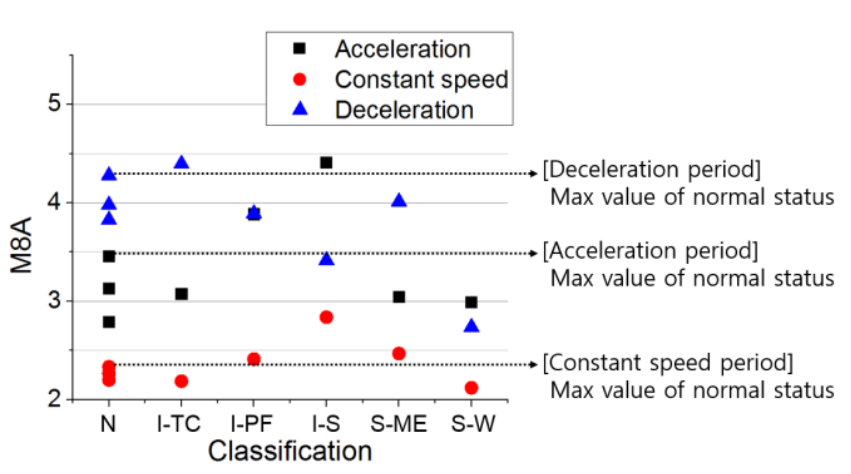

Figure 8. Average of M8A using TSA on the operation test mode

\subsection{Health data optimization}

In order to apply fault diagnostic of gearboxes based on vibration signals in construction machinery, several methods used in other industries have been applied. Although the peaks due to the fault were identified on the time domain signal, the accuracy of the fault diagnostic due to the peakbased feature was confirmed to be lower than expected. In addition, frequency-based methodologies have been found to be limited in use at constant speed conditions. The application of AR-MED and Spectral Kurtosis is considered to have a lot of limitations because it is meaningless to carry out fault diagnostic only considering constant speed conditions in excavator operating conditions. In the case of TSA, since rotational speed information is used, there is an advantage that it can be used in the transient speed condition, but the fault diagnostic performance in this study is not good.

Nonetheless, in order to perform a fault diagnostic of a planetary gearbox in an excavator, the performance of the TSA induced features that can be processed in the excavator controller and detectable in the transient speed mode should be improved. The vibration signal obtained from the excavator is shown in Eq. (4) are assumed to be approximately four components: vibrations related to rotation of the diagnostic target $r(t)$, noise sources coming from excavator $n_{v}(t)$, random noisy signals with unknown sources $n_{u}(t)$, and noisy signals due to the circumstances of the equipment $n_{c}(t)$.

$$
v(t)=r(t)+n_{v}(t)+n_{u}(t)+n_{c}(t)
$$

Conventional signal processing methodologies focus on eliminating the rotation signal, denoising and emphasizing the peak signal without considering the part of the noise source that varies depending on the environment of the equipment. However, in the gearbox of a construction 
machinery, the amplitude of the vibration signal collected from the gearbox is too small and signals due to the environment or operation of the equipment are quite dominant. Therefore, in this study, a health data (NKA, Normalized Kurtosis using interval Averaging) like Eq. (5) is proposed.

$$
\mathrm{NKA}=\log (\text { Health data }) *\left(N_{a}+0.01\right)
$$

where Health data refers to the FM4, M6A, and M8A used in this study. $N_{a}$ means a value rescaled through min-max normalization as shown in Eq (6). To prevent health data from being multiplied by 0 , add a correction factor of 0.01 .

$$
\mathrm{N}_{\mathrm{a}}=\frac{E-E_{\text {min }}}{E_{\text {max }}-E_{\text {min }}}
$$

where $E$ is the average value of the data points for obtaining the health data. $E_{\min (\text { or } \max )}$ means the minimum or maximum average value of $E$.

Considering the physical meaning of Eq (5). in Eq (4)., noise sources other than $r(t)$ are greatly reduced through TSA. At this time, since the signal due to fault is included in the rotation signal, health data for peak detection such as M8A is used. However, in equipment such as an excavator, the ratio of $n_{c}(t)$ occupied by a noise source is very large. Therefore, it is considered that the effect of signals other than $r(t)$ is diluted in each signal range in the result of normalization and multiplication. The results of classification of normal and fault of TSA using NKA are shown in Fig 9. It is confirmed that the minimum value of the fault is larger than the maximum value of the normal in all the periods of acceleration, constant speed and deceleration. The distribution of health data for each test case is shown in Figure 10 regardless of the speed condition. Steady-type mechanical errors have some overlapping sections, but they are completely separated for all other faulty cases.

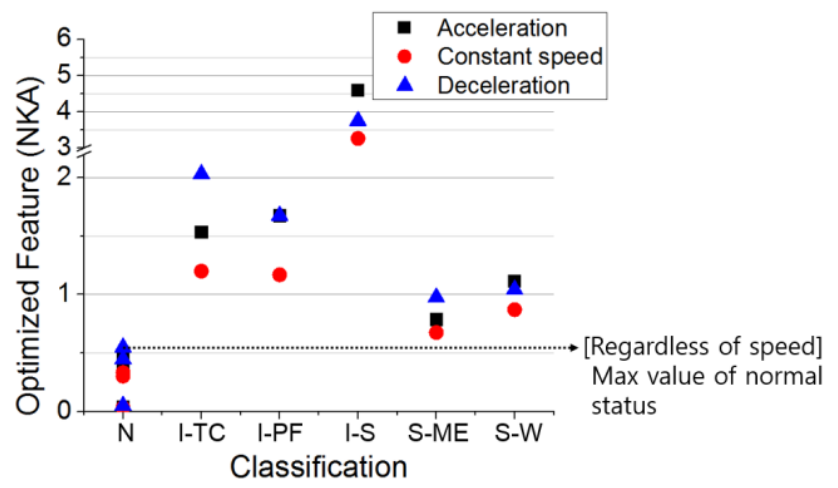

Figure 9. Average of optimized feature using TSA

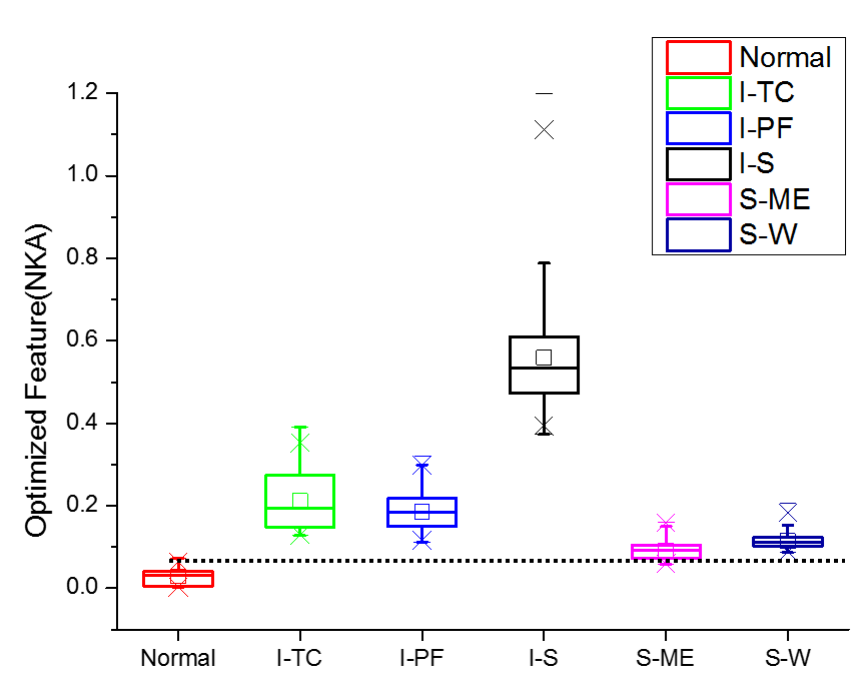

Figure 10. Box-plot of optimized feature using TSA

\section{CONClusions AND FUTURE WORK}

The purpose of this study is to empirically diagnose the failure of gears mainly occurring in gearbox system of construction machinery:

1. Through the field case studies, the system to be diagnosed and five representative faults were selected and utilized as arbitrary-reproduced faults in the actual vehicle experiments.

2. Under the condition that the gear box rotates at a constant speed, it has been confirmed that it can distinguish several faults by three methods of AR-MED, Spectral Kurtosis and TSA.

3. When considering the actual operating conditions of the excavator, it was confirmed that only the TSA methodology among the above three methods could be used, and some faults were classified through acceleration and deceleration by signal processing.

4. The Health Index, NKA has been proposed considering the characteristics of the excavator, which is influenced by the working environment, and succeeded in distinguishing faults from the normal state.

This study is the second study for the commercialization of the gearbox fault detection technology on the construction machinery. In the future, it will be further intensified with studies such as sampling rate and data storage optimization, increase in number of detectable faults and gears through the validation process using the field samples, and reconstruction of gearbox speed information using basic sensor signals in equipment. 


\section{REFERENCES}

Arakawa, S. (2002). Development and Deployment of KOMTRAX STEP 2. Komatsu technical report, 48(150), pp. 8-14.

He, Q. H., He, X. Y., \& Zhu, J. X. (2008). Fault detection of excavator's hydraulic system based on dynamic principal component analysis. Journal of Central South University of Technology, 15(5), 700.

Keon, Kim., (2017). Quantitative performance evaluation of a knock sensor for vibration-based fault diagnosis in a planetary gearbox. Master's thesis. Seoul National University, Seoul, Korea.

Lee, J., Chen, Y., Al-Atat, H., AbuAli, M., \& Lapira, E. (2009). A systematic approach for predictive maintenance service design: methodology and applications. International Journal of Internet Manufacturing and Services, 2(1), 76-94.

Yongzhou Li., Kang Ding., Goulin He., \& Huibin Lin. (2016). Vibration mechanisms of spur gear pair in healty and fault states. Mechanical Systems and Signal Processing, 81, pp. 183-201

Sawalhi, N., Randall, R. B., \& Endo, H. (2007). The enhancement of fault detection and diagnosis in rolling element bearings using minimum entropy deconvolution combined with spectral kurtosis. Mechanical Systems and Signal Processing, vol 21 (6), pp. 2616-2633.

Antoni, J., \& Randall, R. B. (2006). The spectral kurtosis: application to the vibratory surveillance and diagnostics of rotating machines. Mechanical Systems and Signal Processing, 20(2), 308-331.

McFadden, P. D. (1987). Examination of a technique for the early detection of failure in gears by signal processing of the time domain average of the meshing vibration. Mechanical Systems and Signal Processing, vol. 1 (2), pp. 173-183.

Zakrajsek, J. J., Townsend, D. P., \& Decker, H. J., (1993). An analysis of Gear Fault Detection Methods as Applied to Pitting Fatigue Failure Data, The Systems Engineering Approach to Mechanical Failure Prevention, $47^{\text {th }}$ Meeting of MFPG.

Martin, H.R., (1989). Statistical Moment Analysis As a Means of Surface Damage Detection, Proceedings of the $7^{\text {th }}$ International Modal Analysis Conference, Society fur Experimental Mechanics, Schenectady, MY, pp. 10161021.

\section{BIOGRAPHIES}

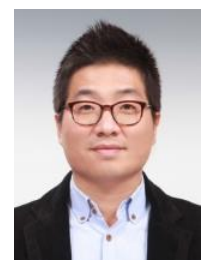

Keon Kim received the B.S. degree from Korea Aerospace University, Goyang-si, South Korea, in 2009, in material science and the M.S degree from the Department of Mechanical and Aerospace Engineering at Seoul National University, Seoul, South Korea, in 2017, in mechanical engineering.
He has been working in Doosan Infracore Co., Ltd., a construction machinery company since 2009 , for materials development, tribology, and PHM. He is currently working on several methodologies for applying PHM to the construction machinery. 